\title{
165.
}

\section{ON SOME GEOMETRICAL THEOREMS RELATING TO A TRIANGLE CIRCUMSCRIBED ABOUT A CONIC.}

[From the Quarterly Mathematical Journal, vol. I. (1857), pp. 169-175.]

THE following investigations were suggested to me by Sir F. Pollock's interesting paper "On a Geometrical Theorem relating to an Equilateral Triangle circumscribed about a Circle," [Quart. Math. Jour. t. I. (1857), pp. 167-169].

If on the sides of a triangle $A B C$, there be taken points $\alpha, \beta, \gamma$, such that $A \alpha, B \beta, C \gamma$ meet in a point $O$; and if on each side of the triangle there be taken two points forming with the two angles on the same side an involution having the first-mentioned point on the same side for a double point; then if three of the six points lie in a line, the two lines are said to be harmonically related with respect to the triangle $A B C$ and point 0 . Call these the lines $(r),(s)$.

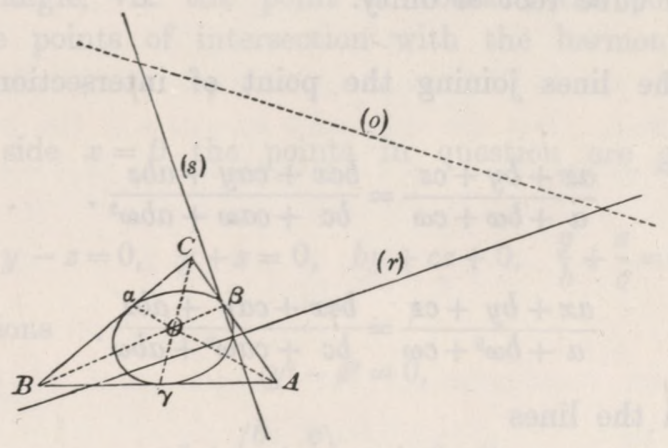

The triangle $A B C$ and point $O$ give rise to a determinate conic; viz., the conic touching the sides at the points $\alpha, \beta, \gamma$. 
The harmonic of the point $O$ with respect to the triangle is a line (o), which is also the polar of $O$ with respect to the conic. The conic meets this line in two points (as the figure is drawn, imaginary ones), $I, J$.

Suppose now that the harmonic lines $(r),(s)$ are harmonically related to the points $I, J$, (i.e. let the lines $(r),(s)$ and the lines through their point of intersection and $I$, $J$, be a harmonic pencil, (or, what is the same thing, let $I, J$, and the points in which the line of junction meets the lines $(r),(s)$ be a harmonic range), then, Theorem, the point of intersection of the lines $(r),(s)$ lies on the conic.

In order to prove this, take

$$
\begin{aligned}
& x=0, y=0, z=0, \text { for the equations of } B C, C A, A B, \\
& x=y=z, \text { for the point } O,
\end{aligned}
$$

the equations of the harmonic lines will be

$$
\begin{aligned}
& a x+b y+c z=0, \\
& \frac{x}{a}+\frac{y}{b}+\frac{z}{c}=0,
\end{aligned}
$$

the equation of the conic is

$$
x^{2}+y^{2}+z^{2}-2 y z-2 z x-2 x y=0 ;
$$

the equation of the line $(0)$ is

$$
x+y+z=0 .
$$

The coordinates of the points $I, J$, are

$$
x: y: z=1: \omega: \omega^{2},
$$

and

$$
x: y: z=1: \omega^{2}: \omega,
$$

where $\omega$ is an imaginary cube root of unity.

The equations of the lines joining the point of intersection of $(r),(s)$ with the points $I, J$, are

$$
\frac{a x+b y+c z}{a+b \omega+c \omega}=\frac{b c x+c a y+a b z}{b c+c a \omega+a b \omega^{2}},
$$

and

$$
\frac{a x+b y+c z}{a+b \omega^{2}+c \omega}=\frac{b c x+c a y+a b z}{b c+c a \omega^{2}+a b \omega},
$$

and these will form with the lines

$$
\begin{aligned}
& a x+b y+c z=0, \\
& b c x+c a y+a b z=0,
\end{aligned}
$$


a harmonic pencil, if

i.e., if

$$
\begin{gathered}
\left(a+b \omega+c \omega^{2}\right)\left(b c+c a \omega^{2}+a b \omega\right) \\
+\left(a+b \omega^{2}+c \omega\right)\left(b c+c a \omega+a b \omega^{2}\right)=0
\end{gathered}
$$

$$
6 a b c-b c^{2}-b^{2} c-c a^{2}-c^{2} a-a b^{2}-a^{2} b=0 ;
$$

which is, therefore, the condition in order that $(r),(s), I, J$ may be harmonically related.

The coordinates of the point of intersection of $(r),(s)$ are

$$
x: y: z=a\left(b^{2}-c^{2}\right): b\left(c^{2}-a^{2}\right): c\left(a^{2}-b^{2}\right) ;
$$

and substituting these values in

$$
x^{2}+y^{2}+z^{2}-2 y z-2 z x-2 x y,
$$

we see, $\grave{a}$ priori, that the result contains the factor

$$
2 a b c+b c^{2}+b^{2} c+c a^{2}+c^{2} a+a b^{2}+a^{2} b=(b+c)(c+a)(a+b) ;
$$

in fact $b+c=0$ gives $x: y: z=0: b\left(b^{-}-a^{2}\right): b\left(b^{2}-a^{2}\right)$, i.e. $x=0, y-z=0$, which makes $x^{2}+y^{2}+z^{2}-2 y z-2 z x-2 x y$ vanish; and so for the other factors.

Effecting the substitution, we find

$$
\begin{aligned}
x^{2}+y^{2}+z^{2}-2 y z-2 z x-x y=\left(2 a b c+b c^{2}+\right. & \left.b^{2} c+c a^{2}+c^{2} a+a b^{2}+a^{2} b\right) \\
& \times\left(-6 a b c+b c^{2}+b^{2} c+c a^{2}+c^{2} a+a b^{2}+a^{2} b\right),
\end{aligned}
$$

which equals 0 , on account of the second factor.

Hence the point of intersection of the lines $(r),(s)$ lies upon the conic. Q.E.D.

Consider, next, a side of the triangle: then, Theorem,- the following four points on a side of the triangle, viz. the point of contact, the point of intersection with the line $(o)$, and the points of intersection with the harmonic lines $(r),(s)$, form a harmonic range.

In fact, for the side $x=0$ the points in question are given by means of the equations

$$
y-z=0, \quad y+z=0, \quad b y+c z+0, \quad \frac{y}{b}+\frac{z}{c}=0,
$$

and forming the equations

$$
\begin{gathered}
y^{2}-z^{2}=0 \\
y^{2}+\left(\frac{b}{c}+\frac{c}{b}\right) y z+z^{2}=0
\end{gathered}
$$

the theorem is at once seen to be true. Q.E.D. 
It is interesting to give the analytical expressions for some other of the points and lines of the figure. The harmonic lines $(r),(s)$, meet in a point of the conic given by the equations

$$
\xi: \eta: \zeta=a\left(b^{2}-c^{2}\right): b\left(c^{2}-a^{2}\right): c\left(a^{2}-b^{2}\right) .
$$

This point may be spoken of as the common point of intersection of the harmonic lines with the conic. The foregoing values give

$$
\begin{aligned}
& \xi^{2}-\eta^{2}-\zeta^{2}=\eta \zeta\left(\frac{b}{c}+\frac{c}{b}\right) \\
& \eta^{2}-\zeta^{2}-\xi^{2}=\zeta \xi\left(\frac{c}{a}+\frac{a}{c}\right) \\
& \zeta^{2}-\xi^{2}-\eta^{2}=\xi \eta\left(\frac{a}{b}+\frac{b}{a}\right)
\end{aligned}
$$

which may also be written

$$
\begin{aligned}
& \xi^{2}-(\eta-\zeta)^{2}=\eta \zeta \frac{1}{b c}(b+c)^{2}=Q R \\
& \eta^{2}-(\zeta-\xi)^{2}=\zeta \xi \frac{1}{c a}(c+a)^{2}=R P, \\
& \zeta^{2}-(\xi-\eta)^{2}=\xi \eta \frac{1}{a b}(a+b)^{2}=P Q
\end{aligned}
$$

if for shortness

$$
\begin{gathered}
-\xi+\eta+\zeta=P, \\
\xi-\eta+\zeta=Q, \\
\xi+\eta-\zeta=R,
\end{gathered}
$$

formulæ which will be presently useful.

Each harmonic line meets the conic in another point, which may be termed the simple point of intersection. For the line $a x+b y+c z=0$, the coordinates of the simple point of intersection are given by $x: y: z=\eta \zeta(b+c)^{2}: \zeta \xi(c+a)^{2}: \zeta \eta(a+b)^{2}$; or, what is the same thing, by

$$
x: y: z=\frac{1}{P a}: \frac{1}{Q b}: \frac{1}{R c},
$$

and, in like manner, the coordinates of the simple point of intersection of the line $\frac{x}{a}+\frac{y}{b}+\frac{z}{c}=1$ are given by

$$
x: y: z=\frac{a}{P}: \frac{b}{Q}: \frac{c}{R}
$$


Hence the equation of the line joining the two simple points of intersection is

$$
\left|\begin{array}{ccc}
x, & y, & z \\
\frac{1}{P a}, & \frac{1}{Q b}, & \frac{1}{R c} \\
\frac{a}{P}, & \bar{Q}, & \frac{c}{R}
\end{array}\right|=0,
$$

or expanding and reducing

$$
P \xi x+Q \eta y+R \zeta z=0 .
$$

The equation of the tangent to the conic at the common point of intersection is evidently

$$
P x+Q y+R z=0 .
$$

The last-mentioned lines, together with the harmonic lines $(r),(s)$, viz. the lines

$$
\begin{aligned}
& a x+b y+c z=0, \\
& \frac{x}{a}+\frac{y}{b}+\frac{z}{c}=0,
\end{aligned}
$$

may be considered as the sides of an inscribed quadrilateral; the equation of the conic must therefore be expressible in a form in which this is put in evidence; to do this, I first form the equation

$$
(a x+b y+c z)\left(\frac{x}{a}+\frac{y}{b}+\frac{z}{c}\right)=x^{2}+y^{2}+z^{2}-2\left(1-\frac{Q R}{\eta \zeta}\right) y z-\left(1-\frac{R P}{\zeta \xi}\right) z x-2\left(1-\frac{P Q}{\zeta \eta}\right) x y,
$$

which may also be written

where

$$
(a x+b y+c z)\left(\frac{x}{a}+\frac{y}{b}+\frac{z}{c}\right)=x^{2}+y^{2}+z^{2}-2 \lambda y z-2 \mu z x-2 \nu x y,
$$

and then putting

$$
\begin{aligned}
& \lambda=\frac{\eta^{2}+\zeta^{2}-\xi^{2}}{2 \eta \zeta}, \\
& \mu=\frac{\zeta^{2}+\xi^{2}-\eta^{2}}{2 \zeta \xi}, \\
& \nu=\frac{\xi^{2}+\eta^{2}-\zeta^{2}}{2 \xi \eta},
\end{aligned}
$$

$$
\Delta=\xi^{2}+\eta^{2}+\zeta^{2}-2 \eta \zeta-2 \zeta \xi-2 \xi \eta,
$$

an equation which gives

C. III.

$$
\begin{aligned}
\triangle & =P^{2}-4 \eta \zeta \\
& =Q^{2}-4 \zeta \xi \\
& =R^{2}-4 \xi \eta
\end{aligned}
$$


and

$$
-P Q R-8 \xi \eta \zeta=(\xi+\eta+\zeta) \triangle,
$$

it is easy by means of these relations to verify the identical equation

$$
\begin{aligned}
& 3 \xi \eta \zeta\left(x^{2}+y^{2}+z^{2}-2 y z-2 z x-2 x y\right)+\xi \eta \zeta(a x+b y+c z)\left(\frac{x}{a}+\frac{y}{b}+\frac{z}{c}\right) \\
& -(P \xi x+Q \eta y+R \zeta z)(P x+Q y+R z)=\triangle\left\{-\xi x^{2}-\eta y^{2}-\zeta z^{2}+(\xi+\eta+\zeta)(y z+z x+x y)\right\},
\end{aligned}
$$

or, writing for $\Delta$ its value $\Delta=0$, the equation of the conic takes the form

$$
\xi \eta \zeta(a x+b y+c z)\left(\frac{x}{a}+\frac{y}{b}+\frac{z}{c}\right)-(P \xi x+Q \eta y+R \zeta z)(P x+Q y+R z)=0,
$$

which is as it should be.

It may be added, that the common point of intersection and the points in which the harmonic lines $(r),(s)$ meet a side of the triangle lie in a conic passing through the points $I, J$, such that with respect to this conic the point of contact is the pole of the line (o). Thus for the side $x=0$ the equation of the conic in question is

where

$$
\theta x(x+y+z)+\left(1-\frac{b}{c}-\frac{c}{b}\right) x^{2}+(b y+c z)\left(\frac{y}{b}+\frac{z}{c}\right)=0
$$

$$
\theta=\frac{a(b-c)^{2}(b+c)}{b c(a-b)(c-a)}=\frac{(b+c)^{2}}{2 b c}
$$

and similarly for the other two conies.

In the case where the triangle is an equilateral triangle and the line $(0)$ is the line at $\infty$, the conic becomes a circle, the points $I, J$, are the circular points at infinity, and lines harmonic in respect to the points $I, J$, become lines at right angles to each other: the foregoing results agree, therefore, with Sir F. Pollock's Theorem. 